\title{
Santé et arrêt de tranche en centrale nucléaire : une étude épidémiologique
}

\author{
M.A. TELLE*1, D. HUEZ**, J.M. NIEDBALA**, J. AUCLAIR*, \\ Y. CHARPAK***, J.P. CANALÈS**, C. DUVERGÉ**, H. FOREST**, \\ M. GÉRONDAL**, P.M. PARIS**, J.C. RENAULT**, L. BOSSEVAIN**, \\ P. BLAISE**, M.C. BLANC**, M. GOLDBERG*
}

Une enquête épidémiologique, initiée en 1989, a été réalisée dans les centrales nucléaires de la vallée de la Loire et du Blayais. Elle porte sur les conditions de travail, l'organisation du travail et leurs liens avec la santé lors des arrêts de maintenance annuels dits arrêts de tranche programmés (ATP). Dans cette étude, transversale, la santé a été essentiellement examinée sous ses aspects psychologiques: anxiété et symptômes dépressifs (échelles de Spielberger et CES-D). Des comparaisons ont été effectuées en et hors arrêt, sur quatre groupes prófessionnels différemment sollicités, chaque sujet étant pris pour son propre témoin. Le test du khi-deux a été utilisé pour les variables qualitatives et le test sur la différence pour les variables quantitatives. On observe en ATP des heures supplémentaires et des horaires atypiques déclarés plus fréquents $(p<$ $0,01)$, un rythme de travail et des règles de sécurité jugés plus contraignants, une exposition à l'irradiation jugée plus importante $(p<0,01)$. Des modifications de l'état psychologique en ATP ont été objectivées pour les agents de conduite alors qu'elles étaient attendues pour les agents de maintenance. Des résultats analogues ont été obtenus en tenant compte de la fréquence des arrêts. Des interprétations sont données en faisant référence à des études qualitatives sur le sujet. Ceci invite à prolonger la réflexion selon les deux axes d'approches, quantitatif et qualitatif.

ABSTRACT An epidemiological survey, started in 1989, was carried out at the nuclear power plants in the Loire river valley and at Le Blayais (France). Working conditions, work organisation and their impact on health during annual maintenance outages were studied. The main areas covered in this cross-sectional study were : anxiety and symptoms of depression using the Spielberger and CES-D scales. Comparisons were made during both a scheduled outage and in normal operation on four distinct groups of workers, each individual being his own control. $A$ chi-square test was used for the quantitative variables and a test on differences for the quantitative variables. During a unit outage, more frequent overtime and

* EDF-GDF, Service général de médecine du travail, Division épidémiologique, 30 avenue de Wagram, 75008 Paris.

** Services de médecine du travail, EDF, CNPE Blayais, Belleville, Chinon, Dampierre, Saint-Laurent. *** Société EVAL, Paris.

1) Adresse actuelle : Institut de protection et de sûreté nucléaire, Département de protection de la santé de l'homme et de dosimétrie (IPSN/DPHD/SEGR), Laboratoire d'épidémiologie et d'analyse du détriment sanitaire (LEADS), BP 6, 92265 Fontenay-aux-Roses Cedex. 
atypical working hours were reported $(p<0.01)$; working rhythms and safety rules were felt as more restrictive and exposure to radiation higher $(p<0.01)$. Detrimental modifications of anxiety and symptoms of depression were observed on controllers whereas expected on maintenance agents. Similar results were observed when considering the rates of outages. Possible readings are given with reference to qualitative studies carried out on this topic, which implies extending our research with both the quantitative and qualitative approaches.

\section{Introduction}

Les réacteurs des centrales nucléaires de type réacteur à eau sous pression (REP) sont arrêtés au moins une fois par an pour des opérations de maintenance industrielle. Les périodes d'arrêt, d'une durée moyenne de cinq à six semaines, sont programmées et qualifiées d'arrêt de tranche programmé : $A T P$. Elles engendrent pour les agents concernés une surcharge de travail accompagnée d'une modification de l'organisation et des conditions de travail. Les travaux effectués pendant ces périodes sont fondamentaux pour le maintien d'une sûreté optimale de l'installation.

Pour mieux appréhender les caractéristiques de ces arrêts et évaluer leurs conséquences en termes de santé, une étude épidémiologique a été entreprise. Elle décrit les arrêts en termes de conditions de travail et d'organisation réelle du travail et aborde la santé essentiellement sous ses aspects psychologiques: anxiété et symptômes dépressifs.

\section{Population et méthode}

La population étudiée est constituée de l'ensemble des agents des centrales de la vallée de la Loire et du Blayais, soit environ 4500 personnes, à l'exception des agents ayant participé aux tests préalables à l'enquête et du personnel des services médicaux. L'effectif important de la population et le temps nécessaire pour remplir le questionnaire (une heure environ) ont conduit à procéder par échantillonnage. Un tirage au sort au sixième a été effectué. L'échantillon définitif, compte tenu des refus et des absences pour congés ou maladie au moment des convocations, est constitué de 565 agents sur les 700 déterminés par tirage au sort.

Les agents ont été répartis en quatre groupes pour les raisons suivantes: d'une part, les arrêts n'affectent pas tous les agents de la même façon. Le personnel de maintenance est très sollicité (préparation de travaux, suivi d'entreprises extérieures, délais stricts à respecter...) alors qu'une partie du personnel administratif n'est pas du tout concernée. D'autre part, pour certaines catégories d'agents, l'activité revêt des caractéristiques particulières dont on sait par la littérature qu'elles ont des conséquences sur la santé. Il s'agit notamment de l'organisation en $3 \times 8$ des horaires de travail [2]. 
La répartition adoptée est la suivante : maintenance lourde (ensemble des sections opérationnelles très présentes sur le terrain et dont l'activité est fortement modifiée en période d'arrêt) ; fonctionnel gestion (autres agents sollicités par les arrêts mais plutôt sur des aspects fonctionnels et de gestion) ; conduite (exploitation $3 \times 8$ et ingénieurs de sûreté - radioprotection dits ISR); autres (dits "SUG autres" pour sous-unité de gestion : autres agents non compris dans les catégories précédentes, essentiellement administratifs).

Enfin, pour tenir compte de la fréquence des arrêts programmés auxquels participent les agents dans l'année, deux autres sous-ensembles d'agents ont été définis: (i) les agents des sous-unités techniques (SUT), structures qui existent sur les sites ayant deux paires de tranches, soit quatre réacteurs. Elles regroupent tous les agents de maintenance pouvant participer à quatre arrêts dans l'année (un par tranche). Il s'agit de mécaniciens, chaudronniers, électriciens, agents des services généraux. Y sont aussi rattachés des agents des services fonctionnels associés aux SUT (radioprotection, préparation, gestion d'arrêt...) ; (ii) les agents des sous-unités centrales (SUC) hors conduite (exclus par leur activité spécifique) qui sont rattachés à une paire de tranche précise et ne peuvent donc participer qu'à deux arrêts de tranche programmés par an.

Il s'agit d'une enquête prospective: on observe, en effet, chaque sujet à deux moments différents : en et hors arrêt. Deux contraintes ont dû être respectées dans la définition des périodes en arrêt et hors arrêt. Il fallait d'une part que les agents soient en arrêt de tranche depuis quelque temps pour qu'apparaissent d'éventuels symptômes d'anxiété ou de dépression et d'autre part que les périodes en et hors arrêt soient suffisamment éloignées les unes des autres pour que l'on puisse les caractériser sans ambiguïté. La définition suivante a donc été retenue : en arrêt de tranche, période commençant trois semaines après l'arrêt du réacteur et se terminant au plus tard une semaine après la fin de l'arrêt; hors arrêt de tranche, période commençant deux mois après la fin de l'arrêt et se terminant un mois avant le début de l'arrêt suivant. Pour éviter un effet éventuel de l'ordre de passation du questionnaire, l'échantillon a été séparé en deux groupes par tirage au sort, le premier (vague A) remplissait le questionnaire d'abord en arrêt puis hors arrêt, le second (vague B) le remplissait d'abord hors arrêt puis en arrêt.

Le recueil des données a duré 22 mois. Il a eu lieu dans les services médicaux, sous la responsabilité du médecin du travail. Chaque sujet a rempli un autoquestionnaire sur les facteurs socioprofessionnels, l'arrêt tel que le perçoit l'agent par rapport à son activité, l'organisation temporelle du travail, les conditions et relations de travail (rythme, autonomie, communications...), la vie sociale et familiale et, enfin, la pathologie générale.

Les questions sur les exigences et les conditions de travail sont des évaluations subjectives des agents sur une échelle de 1 (réponse négative) à 6 (réponse positive).

L'échelle d'anxiété utilisée provient du "State and trait anxiety inventory" (STAI) élaboré par Spielberger [9]. La version actuelle, forme Y, comprend 40 questions, 20 appréhendant l'état d'anxiété (state anxiety inventory) et 20 
référant plutôt aux tendances à l'anxiété (trait anxiety inventory). Nous nous sommes restreints ici à l'état d'anxiété car il réfère à quelque chose de transitoire et susceptible de se reproduire si les conditions qui l'ont provoqué sont à nouveau réunies, alors que le concept de tendance à l'anxiété renvoie à des traits de caractère spécifiques à chaque individu [10]. Nous appellerons ces vingt questions sur l'état d'anxiété "échelle d'anxiété de Spielberger". La version française a été réalisée par Bruchon-Schweitzer à l'Université de Bordeaux II. Le score d'anxiété de Spielberger a été calculé. Il est égal à la somme des réponses après correction d'échelle pour les questions cotées dans un sens opposé à celui de l'anxiété. Il est calculé pour tout individu ayant répondu à au moins 18 questions sur 20 et est alors pondéré par le nombre de réponses. L'échelle variant de 1 à 4 , le score varie de 20 à 80 .

L'échelle de dépressivité CES-D (Center for epidemiologic studies - depression scale) comprend aussi vingt questions. Elle teste des symptômes dépressifs qui peuvent être soit transitoires, soit le reflet d'états dépressifs plus graves. La version française a été mise au point et validée par Fuhrer [5]. Le score de dépressivité CES-D est calculé de manière analogue au score de Spielberger. Les réponses sont prises en compte lorsque l'individu a répondu à au moins 16 questions sur 20 . L'échelle variant de 0 à 3 , le score varie de 0 (dépressivité minimale) à 60 (dépressivité maximale). Trois variables ont été ajoutées pour appréhender de façon subjective l'état de santé général, la fatigue mentale et la forme physique et psychique. Elles sont codées de 1 (réponse péjorative) à 6 .

Les analyses statistiques ont porté à la fois sur l'ensemble des agents et sur chaque sous-groupe au moyen d'analyses appariées confrontant les résultats en arrêt et hors arrêt. On a obtenu ainsi des différences de conditions de travail et de santé en et hors arrêt pour chaque sous-groupe et l'on a pu examiner ensuite pour quels groupes on obtenait les écarts les plus importants entre les deux périodes. Les test utilisés sont le test sur la différence pour les variables quantitatives et le test du khi-deux apparié pour les variables qualitatives. Ils peuvent, en effet, tenir compte des réponses discordantes pour un même agent selon sa situation en ou hors arrêt. Les tests ont été réalisés de façon bilatérale.

Une régression linéaire multiple a été effectuée sur les réponses des agents en arrêt de tranche pour tenter d'expliquer les variables de santé par les variables de conditions de travail. Celle-ci consiste à introduire, pas à pas, les variables de conditions de travail, en ne conservant à chaque étape que les variables explicatives (le seuil étant fixé à 5 ou $10 \%$ ). Quatre variables ont été imposées dans le modèle (c'est-à-dire prises en compte systématiquement): l'ordre de passage du questionnaire, le sexe, l'âge et le niveau hiérarchique.

\section{Résultats}

La représentativité de l'échantillon a été vérifiée. La répartition par âge, sexe, situation familiale, collège (niveau hiérarchique) et ancienneté dans l'entreprise n'est pas statistiquement différente de la population étudiée. 


\subsection{Caractéristiques socio-professionnelles (Tab. I)}

L'échantillon comprend $9 \%$ de femmes appartenant en grande majorité au groupe "SUG autres". Celles-ci n'ont pas été retirées de l'échantillon. En effet, le seul risque pris en faisant ce choix était d'obtenir des résultats biaisés par l'effet connu d'une plus grande déclaration des symptômes d'anxiété et de dépression chez les femmes. Le biais ne pouvait qu'infléchir les résultats vers une surestimation des problèmes de santé dans le groupe "SUG autres". Ce groupe, essentiellement composé de personnel administratif, était par hypothèse le moins affecté par les arrêts. Le biais allait donc "dans le bon sens", c'est-à-dire ne pouvait pas conduire à confirmer l'hypothèse à tort.

La moyenne d'âge est de 35 ans, un peu moins chez les agents de conduite ( 33 ans) et un peu plus dans le groupe "SUG autres" ( 37 ans). Les agents d'exécution sont plus nombreux parmi les agents de maintenance lourde (36\%

TABLEAU I

Répartition, pour chaque groupe professionnel étudié, des agents des centrales nucléaires de la vallée de la Loire et du Blayais par sexe et collège (échantillon tiré au sort d'un sixième de la population)

- ancienneté moyenne pour chaque sous-groupe

Distribution of workers from the nuclear power plants (NPPs)

in the Loire valley and at Le Blayais (France), for each professional group studied, by sex and by job category (sample drawn at random from a sixth of the population) - average seniority for each sub-group

\begin{tabular}{|c|c|c|c|c|c|}
\hline Pros & $\begin{array}{l}\text { nenters } \\
\text { ntena } \\
\text { ourde } \\
\text { stris }\end{array}$ & Ection & ondui & Autr & Tolal \\
\hline$v^{\circ}$ & 210 & 154 & 94 & 107 & 565 \\
\hline - Hommes $(\%)$ & 100 & 94,2 & 98,9 & 62,6 & 91,2 \\
\hline - Femmes $(\%)$ & 0 & 5,8 & 1,1 & 37,4 & 8,8 \\
\hline - Collège & & & & & \\
\hline Exécution (\%) & 36,2 & 17,5 & 18,1 & 22,4 & 25,5 \\
\hline Maîtrise (\%) & 59,5 & 66,2 & 69,1 & 58,9 & 62,8 \\
\hline Cadres (\%) & 4,3 & 16,2 & 12,8 & 18,7 & 11,7 \\
\hline- Age $^{2}$ & 34,7 & 35,7 & 33,5 & 37,4 & 35,3 \\
\hline $\begin{array}{l}\text { - Ancienneté } \\
\text { dans le poste }\end{array}$ & 3,7 & 3,5 & 3,4 & 3,7 & 3,6 \\
\hline $\begin{array}{l}\text { - Ancienneté } \\
\text { sur le site }\end{array}$ & 6,5 & 6,2 & 7,5 & 6,9 & 6,6 \\
\hline - Ancienneté EDF ${ }^{2}$ & 9,9 & 11,0 & 11,5 & 12,8 & 11,0 \\
\hline
\end{tabular}

1 : ISR : ingénieur "sûreté-radioprotection"; SUG : sous-unité de gestion.

2 : moyenne. 
contre 18 à $20 \%$ dans les autres groupes). L'ancienneté est assez semblable dans les différents groupes : dans le poste, elle est de trois ans et demi environ; dans le site, elle est de six ans et demi (un peu plus pour les agents de conduite) et dans l'entreprise, elle est de onze ans (un peu plus dans le groupe "SUG autres").

\subsection{Organisation temporelle et horaires de travail}

Le tableau II met en évidence les différences observées en matière d'organisation temporelle du travail.

- Les horaires particuliers sont plus fréquents en arrêt de tranche : en arrêt, $27,3 \%$ des agents ont travaillé au moins une fois en horaire du matin contre $21,6 \%$ hors arrêt $(p<0,01)$, dans les trois semaines précédant le remplissage du questionnaire. Le contraste est particulièrement marqué pour les agents de maintenance lourde qui travaillent plus souvent en horaire du matin $(14,3 \%$ contre $4,3 \%, p<0,01)$ et un peu plus en horaire de nuit $(4,8 \%$ contre $1 \%$, $p<0,05)$.

\section{TABLEAU II}

Comparaison de l'organisation temporelle du travail en et hors arrêt de tranche programmé pour les agents des centrales nucélaires de la vallée de la Loire et du Blayais

Comparison of working hours management during scheduled outages and in normal operation for workers from the NPPs in the Loire valley and at Le Blayais, France

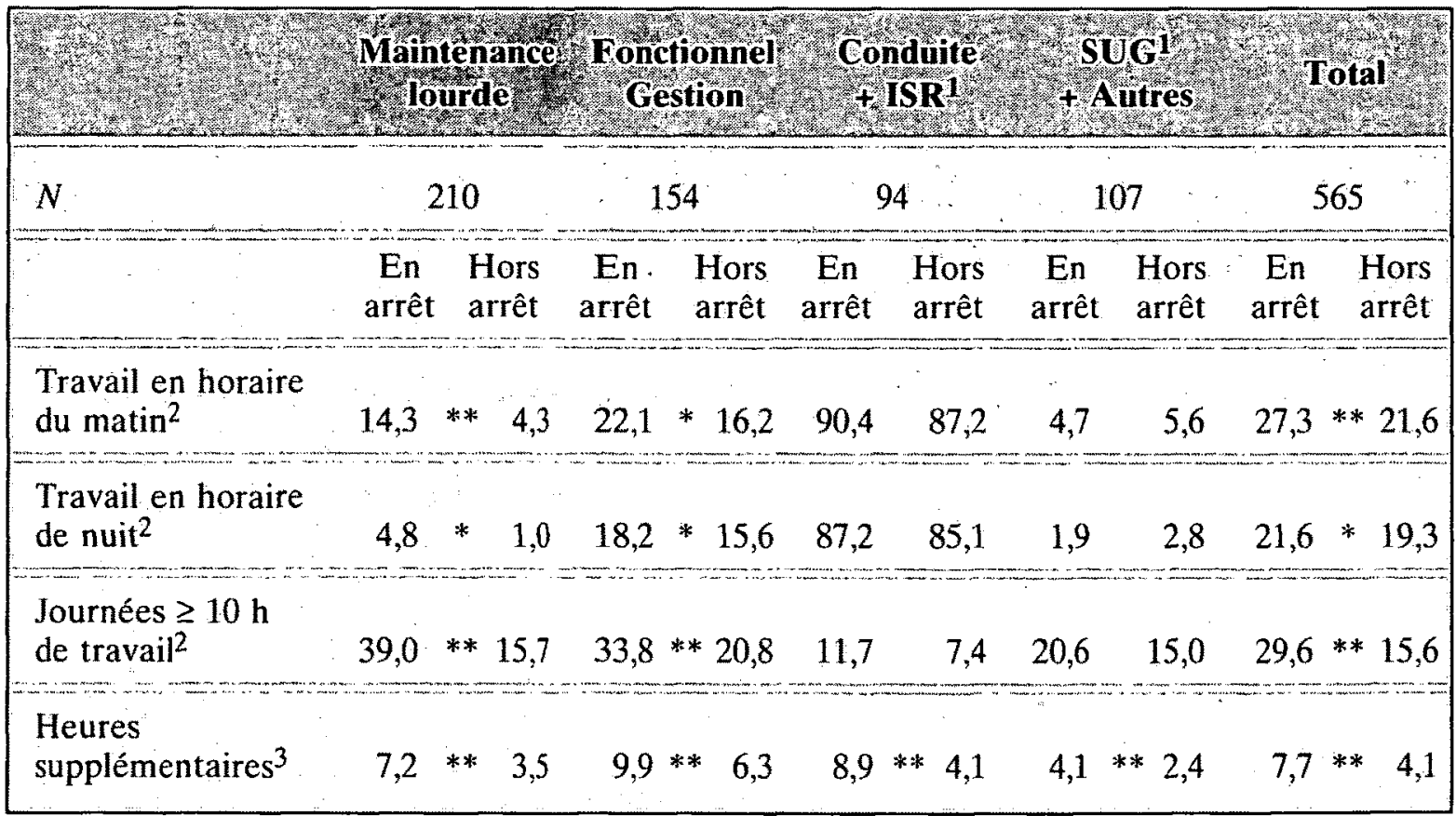

1 : ISR : ingénieur "sûreté-radioprotection"; SUG : sous-unité de gestion.

2 : pourcentage ; $3:$ moyenne.

* $p<0,05 ;{ }^{* *} p<0,01$. 
- Les longues journées de travail sont plus fréquentes en arrêt : 29,6\% des agents ont eu au moins une journée de travail de plus de $10 \mathrm{~h}$ en arrêt contre $15,6 \%$ hors arrêt, $p<0,01$. Là encore le contraste est plus fort pour les agents de maintenance lourde (39\% contre $15,7 \%, p<0,01)$. Pour les agents du groupe "Fonctionnel Gestion" il est de 33,8\% contre 20,8\% $(p<0,01)$.

- Les heures supplémentaires sont plus importantes pour tous les groupes en arrêt : $7,7 \mathrm{~h}$ en moyenne contre $4,1 \mathrm{~h}(p<0,01)$. Le groupe "SUG autres" est le moins affecté $(4,1 \mathrm{~h}$ contre $2,4 \mathrm{~h}, p<0,01)$.

\subsection{Exigences et conditions de travail (Tab. III)}

En arrêt, pour chaque sous-groupe d'agent, le rythme de travail est jugé plus imposé $(3,2$ contre $3,8, p<0,01)$ et plus insupportable $(4,5$ contre 5 , $p<0,01)$. Pour tous les sous-groupes sauf pour le groupe "SUG autres", il y a plus de contraintes liées à la sécurité $(4,2$ contre $4,5, p<0,01)$ en arrêt et la gêne liée au temps d'attente est plus importante $(3,6$ contre $4,2, p<0,01)$. Enfin, le groupe "conduite" est le seul à mettre en évidence une moins bonne transmission d'informations en arrêt $(3,6$ contre $3,9, p<0,05)$.

Pour les variables psychosociales du type intérêt du travail, autonomie et relations dans le travail, on ne constate pas de différence.

\subsection{Aspects psychologiques}

Le tableau IV synthétise les résultats obtenus pour les variables psychologiques.

Trois questions portaient directement sur l'état psychologique ressenti. Les agents répondaient sur une échelle de 1 (réponse péjorative) à 6 . En arrêt, la moyenne des réponses passe de 3,9 à 3,5 pour la fatigue mentale $(p<0,01)$, de 4,2 à 4 pour la forme physique et psychique $(p<0,01)$ et de 4,1 à 3,8 pour l'anxiété $(\mathrm{p}<0,05)$. Au niveau des sous-groupes, ces différences persistent surtout pour les agents de conduite et, dans une moindre mesure, pour le groupe "Maintenance lourde".

Contrairement aux trois variables précédentes, le score d'anxiété de Spielberger et le score CES-D sont calculés à partir de questions portant sur un ensemble de symptômes abordant le concept étudié, anxiété ou tendances dépressives selon le cas. On observe un surcroit d'anxiété en arrêt de tranche. Il provient des agents de conduite dont le score passe de 32,7 à 37,7, $p<0,01$. Le même phénomène est observé pour les symptômes dépressifs : le score des agents de conduite passe de 9,2 à 11,4 en arrêt, $p<0,01$.

Précisons que le seuil à partir duquel on considère dans la littérature qu'il y a tendance dépressive est de 17 pour les hommes et de 23 pour les femmes; $14 \%$ de l'ensemble des agents de l'échantillon se situent au-dessus de ce seuil en et hors arrêt. Pour les agents de conduite, il passe de $13 \%$ hors arrêt à $20 \%$ en arrêt. 
TABLEAU III

Comparaison des exigences de travail et des conditions de travail en et hors arrêt de tranche programmé pour les agents des centrales nucléaires de la vallée de la Loire et du Blayais -

(valeurs moyennes des scores sur une échelle de 1 à 6)

Comparison of work requirements and working conditions during a scheduled outage and in normal operation for workers from the NPPs in the Loire valley and at Le Blayais, France (mean scores on a scale from 1 to 6)

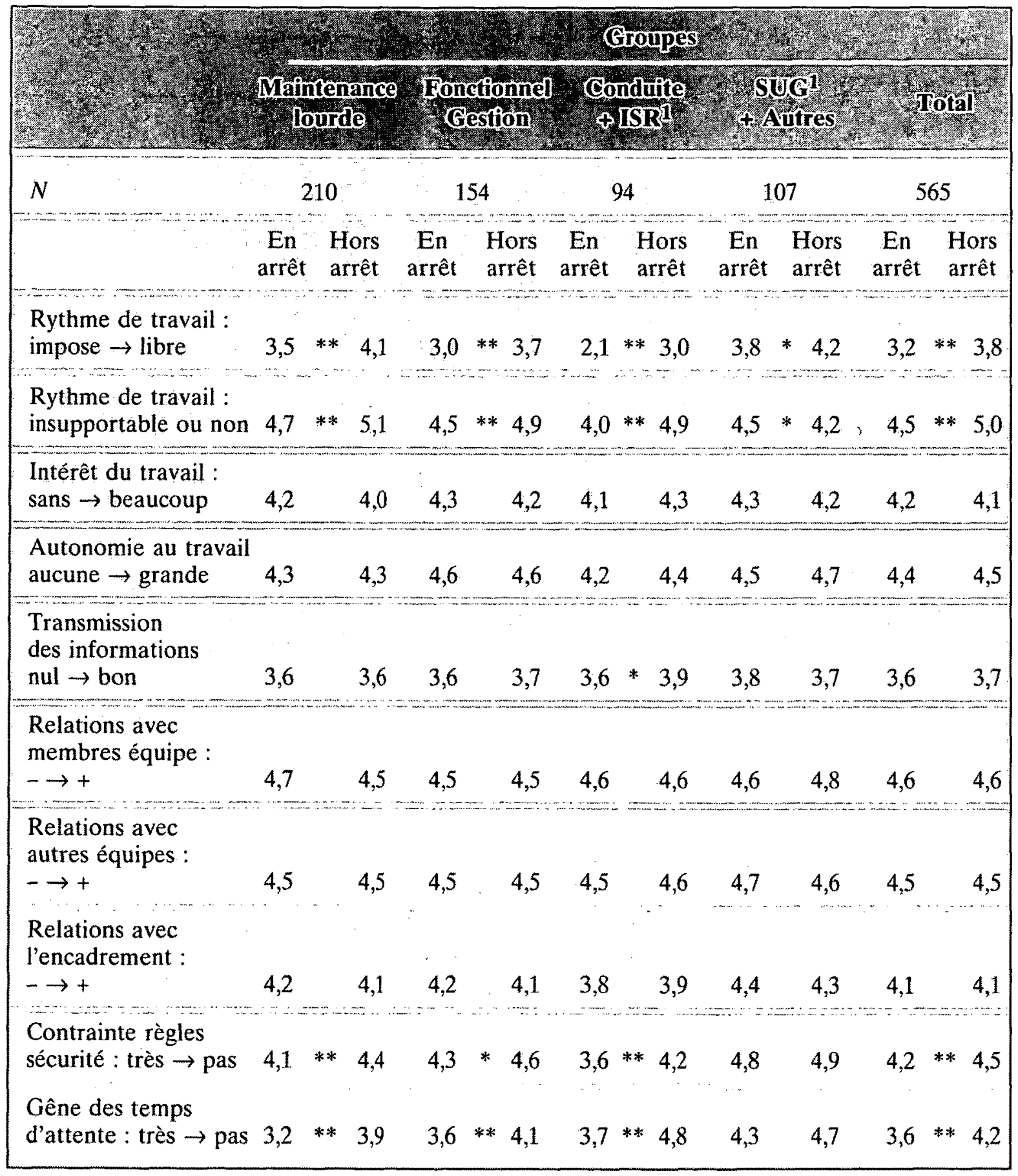

1 : ISR : ingénieur "sûreté-radioprotection"; SUG : sous-unité de gestion.

${ }^{*} p<0,05 ; * * p<0,01$. 


\section{TABLEAU IV}

Comparaison, pour chaque groupe professionnel étudié, de certaines caractéristiques psychologiques en et hors arrêt de tranche programmé des agents des centrales nucléaires de la vallée de la Loire et du Blayais Comparison, for each group of workers studied, of certain psychological characteristics during a scheduled unit outage and in normal operation for workers from NPPs in the Loire valley and at Le Blayais (France)

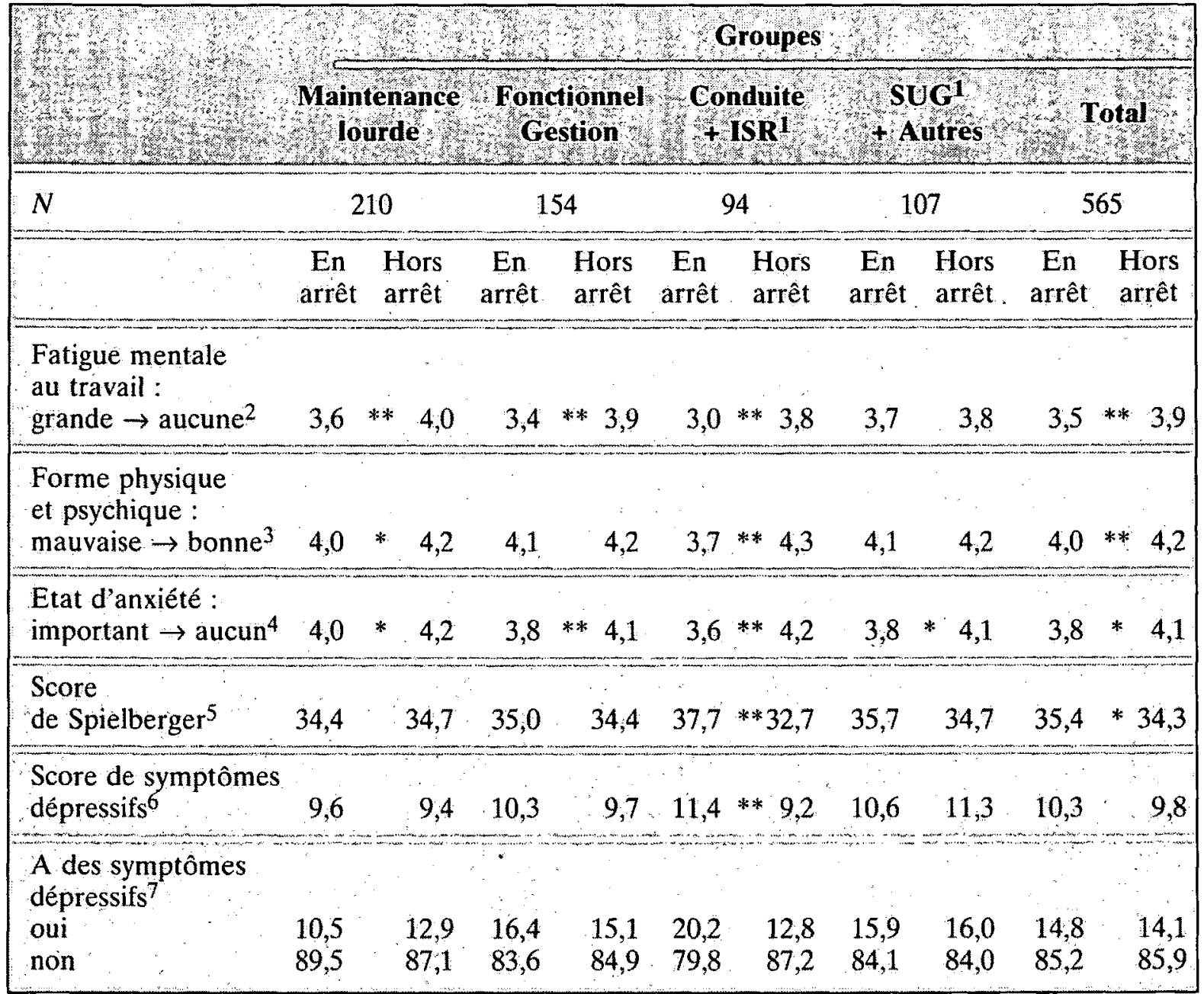

1 : ISR : ingénieur "sûreté-radioprotection"; SUG : sous-unité de gestion.

2 : fatigue ressentie, exprimée sur une échelle de 1 à $6 ; 3:$ forme physique et psychique ressentie, exprimée sur une échelle de 1 à $6 ; 4$ : état d'anxiété ressenti, exprimé sur une échelle de 1 à $6 ; 5:$ somme des scores de 1 à 4 en réponse à l'échelle d'anxiété de Spielberger, pondérée par le nombre de réponse ; 6 : somme des scores de 0 à 3 en réponse à l'échelle de dépressivité CES-D, pondérée par le nombre de réponses; 7 : pourcentage.

${ }^{*} p<0,05 ;{ }^{* *} p<0,01$.

\subsection{Santé et fréquence des arrêts}

Le tableau V montre que les scores du groupe "SUC hors conduite" qui participe à deux arrêts au plus par an augmentent en arrêt (score d'anxiété : de 34,2 à 35,6, $p<0,05$ et score de dépressivité : de 8,9 à 10,1,p<0,05) mais moins que pour les agents de conduite. Pour le groupe "SUT" qui peut être en arrêt jusqu'à 8 mois sur 12 , on ne constate aucune différence. 
TABLEAU $\mathrm{V}$

Comparaison de l'anxiété et de la dépressivité en et hors arrêt de tranche programmé pour les agents des centrales nucléaires de la vallée de la Loire et du Blayais et en fonction de la fréquence des arrêts

Comparison of anxiety and depressive tendencies during a scheduled outage and in normal operation for workers from NPPs in the Loire valley and at Le Blayais (France)

\begin{tabular}{|c|c|c|c|c|c|}
\hline 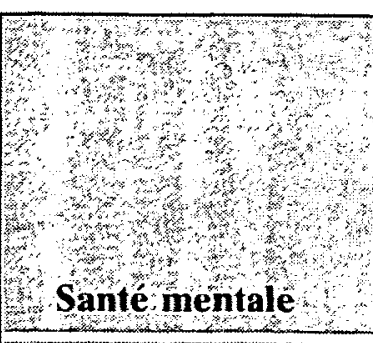 & $\begin{array}{l}\text { Fréquence } \\
\text { des arrêts } \\
\text { Groupe } \\
\text { Situation }\end{array}$ & $\begin{array}{l}4 \\
(N=120) \\
S U T \\
N=120) \\
S\end{array}$ & $\begin{array}{l}2 \\
(N=186) \\
S U C^{1} \\
N=186) \\
\end{array}$ & $\begin{array}{l}0 \\
(N=94) \\
\text { Conduite } \\
(N=94)\end{array}$ & $\begin{array}{l}0 \\
(N=285) \\
\text { Autres } \\
N=285) \\
\end{array}$ \\
\hline \multirow{2}{*}{$\begin{array}{l}\text { Score d'anxiété } \\
\text { (Spielberger) }\end{array}$} & en ATP & 34 & 35,6 & 37,7 & 34,5 \\
\hline & hors ATP & 35,2 & 34,2 & 32,7 & 34,9 \\
\hline \multirow{2}{*}{$\begin{array}{l}\text { Score de dépressivité } \\
\text { (CES-D) }\end{array}$} & en ATP & 9,3 & 10,1 & 11,4 & 10,1 \\
\hline & hors ATP & 9,8 & 8,9 & 9,2 & 8,9 \\
\hline
\end{tabular}

1 : SUC : sous-unité centrale - agents participant à deux arrêts de tranche par an ; SUT : sous-unité technique - agents participant à quatre arrêts de tranche par an.

${ }^{*} p<0,05 ;{ }^{* *} p<0,01$.

L'organisation temporelle et les conditions de travail des groupes SUT et SUC hors conduite sont quasiment les mêmes. Seule la fréquence des périodes d'arrêt et leur durée totale dans l'année les différencient. La comparaison entre les deux groupes pourra donc refléter les effets des variables "fréquence et durée d'arrêt dans l'année" sur les variables psychologiques.

\subsection{Relations entre conditions de travail en arrêt de tranche et santé}

La régression linéaire effectuée pour mettre en évidence les variables déterminantes pour la santé en arrêt de tranche donne les résultats suivants (tableau VI) :

- l'ordre de passage du questionnaire n'est pas lié aux variables de santé (il n'existe pas d'effet passage du questionnaire);

- l'âge et le sexe sont liés aux variables de santé; les femmes et les plus âgés ont des scores de dépressivité plus élevés ;

- l'organisation temporelle du travail n'est quasiment pas liée à la santé, seul le "travail du matin" a un effet pénalisant sur le score d'anxiété $(p<0,05)$;

- la seule variable sur les conditions de travail (ressenties) pour laquelle on trouve une association avec les deux scores (anxiété et dépressivité) est celle qualifiant le rythme de travail d'insupportable (respectivement $p<0,001$ et $p<0,01)$; 


\section{TABLEAU VI}

Relations entre les variables de conditions de travail et de santé en arrêt de tranche programmé mises en évidence par régression linéaire multiple Relations between health variables and working conditions during a scheduled outage shown by multiple linear regression

\begin{tabular}{|c|c|c|}
\hline Whes & nxié & ressivité? \\
\hline Passage & NS & NS \\
\hline Sexe & $\left({ }^{*}\right)$ & $* * *$ \\
\hline Age & $\left({ }^{*}\right)$ & $* *$ \\
\hline Niveau hiérarchique & $(*)$ & NS \\
\hline Variables temporelles & & \\
\hline Horaire matin & NS & $(*)$ \\
\hline Horaire nuit & NS & NS \\
\hline Travail avant $6 \mathrm{~h}$ matin & $*$ & NS \\
\hline Travail $>12 \mathrm{~h} /$ jour & NS & NS \\
\hline Heures supplémentaires & & \\
\hline Variables subjectives & & 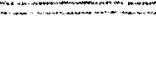 \\
\hline Rythme imposé & NS & NS \\
\hline Rythme insupportable & $* * *$ & $* *$ \\
\hline Intérêt au travail & $* *$ & NS \\
\hline Autonomie au travail & NS & NS \\
\hline Transm. informations & ** & NS \\
\hline Relations voisins travail & NS & **** \\
\hline Relations autres équipes & $* * *$ & NS \\
\hline Relations encadrement & NS & $* *$ \\
\hline Contraintes sécurité & ** & NS \\
\hline Gêne attente & NS & $* * *$ \\
\hline Nombre semaines d'ATP depuis 3 semaines & $(*)$ & $(*)$ \\
\hline Nombre semaines d'ATP depuis 6 semaines & NS & NS \\
\hline
\end{tabular}

NS : non significatif $;\left(^{*}\right): p<0,1 ; *: p<0,05 ;{ }^{* *}: p<0,01 ; * * *: p<0,001$

1 : évaluée par l'échelle d'anxiété de Spielberger ; 2 : évaluée par l'échelle de dépressivité CES-D.

- on note un effet de la qualité de la transmission d'information sur le score de dépressivité ; la détérioration de la qualité de transmission des informations en arrêt de tranche est liée à un score d'anxiété plus élevé $(p<0,01)$;

- enfin, on note une tendance à un lien entre le nombre de semaines d'arrêt de tranche effectuées depuis trois semaines tel qu'il est évalué par l'agent, les scores de Spielberger et le CES-D ; ces scores augmentent avec le nombre de semaines $(p<0,1)$; par contre, le nombre de semaines d'arrêt de tranche depuis 6 semaines n'est pas explicatif. 


\section{Discussion}

L'étude épidémiologique réalisée sur les liens entre arrêts de tranche en centrale nucléaire et santé a permis de faire les constatations suivantes :

- l'organisation temporelle du travail est plus astreignante en arrêt de tranche : horaires de travail particuliers plus fréquents, plus longues journées de travail et heures supplémentaires plus nombreuses, ceci vaut surtout pour les agents de maintenance lourde; on a, par ordre décroissant de perturbations : les agents du groupe "maintenance lourde", suivis de ceux du groupe "fonctionnel gestion", des agents de conduite et enfin de ceux du groupe "SUG autres";

- pour tous les agents le rythme de travail est jugé plus imposé et plus insupportable en arrêt ;

- pour tous les agents, mis à part ceux du groupe "SUG autres", on constate plus de plaintes sur les contraintes liées à la sécurité et sur les temps d'attente;

- pour le groupe conduite, on constate une moins bonne transmission d'informations en arrêt ;

- les agents les plus affectés en matière d'anxiété et de dépressivité sont les agents de conduite;

- le groupe le plus souvent et le plus longtemps en arrêt (SUT) est celui pour lequel on n'observe pas de différence d'anxiété et de dépressivité en et hors arrêt ;

- la régression linéaire effectuée montre que la variable "rythme de travail insupportable" est déterminante pour l'état psychologique, la qualité de la transmission d'informations joue sur le score de dépressivité ainsi que le nombre de semaines d'arrêt depuis 3 semaines; le nombre de semaines d'arrêt depuis 6 semaines, par contre, n'est pas déterminant.

Le rapprochement des résultats sur les conditions de travail et de ceux concernant l'état psychologique des agents soulève des questions : les groupes les plus contraints a priori par les arrêts ne sont pas ceux pour lesquels on observe le plus de détérioration de santé (anxiété ou dépressivité). Ce résultat persiste si l'on tient compte de la fréquence des arrêts puisque l'on a par ordre décroissant de perturbation:

- agents de conduite,

- agents de maintenance pouvant participer au maximum à deux arrêts par an, par an.

- agents de maintenance pouvant participer au maximum à quatre arrêts

La régression linéaire, en plus des résultats classiques qu'elle donne (âge et sexe liés aux variables de santé), fournit deux éléments d'explication partielle :

- les effets de la qualité de la transmission d'information, déterminants pour le score d'anxiété, pourraient avoir joué pour les agents de conduite, les 
seuls à avoir mentionné ce problème en arrêt et aussi les plus atteints en matière d'anxiété à ces périodes ;

- le nombre de semaines d'arrêt de tranche interviendrait sous la forme d'un effet en $U$ : conséquences importantes du nombre de semaines d'arrêt les trois semaines ayant précédé la passation du questionnaire, pas de conséquences apparentes lorsque l'on considère un passé de six semaines; il semble que rien n'apparaîtrait plus au-delà d'un certain seuil temporel.

On peut faire plusieurs hypothèses pour essayer d'interpréter ces phénomènes apparemment contradictoires :

(i) Certains effets des arrêts de tranche sur la santé ne seraient pas décelés par l'enquête pour des raisons liées aux limites de la méthode. Ce serait le cas, par exemple, d'un effet permanent : le groupe le plus soumis aux contraintes des arrêts en temps durant l'année n'aurait pas le temps de récupérer dans les périodes hors arrêts. Puisque l'on compare des différences de santé, elles seraient ici nulles et le reflet d'un effet permanent. On peut aussi envisager des effets ultérieurs des contraintes actuellement supportées par les agents (effets somatiques à long terme uniquement décelables par un suivi des agents).

(ii) La population la plus contrainte a priori aurait des caractéristiques particulières en termes de santé subjective et objective. Par santé subjective nous pensons au fait qu'elle pourrait avoir un référentiel de santé différent. Habituée à de fortes contraintes, elle pourrait, en effet, surestimer son état de santé par rapport aux autres agents. Pour ce qui concerne la santé objective, nous pensons à l'effet classique du travailleur en bonne santé ("Healthy worker effect") : les agents les plus contraints supporteraient mieux leurs contraintes de travail parce qu'ils ont été sélectionnés sur des critères d'aptitude précis plus ou moins explicites.

(iii) Le groupe le plus contraint conserve réellement un bon état psychologique, et la question subsidiaire devient : par quels mécanismes cela se fait-il ? Y a t-il des mécanismes de défense adaptatifs au sens développé par Dejours [4]: modifications des modes opératoires, écart entre travail réel et travail prescrit plus important, désengagement professionnel... Les résultats d'études de psychodynamique du travail réalisées assez récemment sur des sites nucléaires iraient dans ce sens là [6]. Est-ce un effet du "professionalisme" ? Les agents intervenant sur quatre arrêts de tranche répètent plus souvent les gestes de leur métier dans l'année. Le sentiment de compétence qui peut alors se développer serait propice à une bonne santé [1].

Enfin les résultats sur le surcroît d'anxiété observé chez les agents de conduite méritent aussi réflexion. L'organisation de l'arrêt de tranche luimême et toutes les zones d'incertitude qui lui sont liées pourraient être générateurs d'angoisse et d'anxiété pour les "acteurs opérationnels" s'ils ont le sentiment de ne pas maîtriser suffisamment le déroulement de l'arrêt dans sa globalité. Une partie des enseignements que l'on peut tirer d'études récentes sur l'organisation des arrêts de tranche et leur lien avec la sûreté semblent aller dans ce sens $[1,8]$. 
Deux éléments peuvent intervenir dans le surcroît d'anxiété observé : d'une part, des problèmes liés à une représentation morcelée de l'organisation de l'arrêt de tranche et d'autre part, des problèmes du type "double contrainte" liés à des changements de planning, des pressions diverses (ceci est notamment valable pour les agents de consignation) [6-7]. Pour les agents de conduite, l'incertain est plus fort qu'en conduite normale, le nombre de configurations possibles de l'installation est au moins centuplé; ainsi le travail d'analyse des informations nécessaire à la maîtrise de l'état de l'installation est plus complexe [1]. Le fait que la variable "transmission d'information" soit une variable explicative du score d'anxiété dans la régression linéaire décrite plus haut est tout à fait cohérent avec cette interprétation.

\section{Conclusion}

Cette étude épidémiologique a mis en évidence des problèmes de conditions de travail et de santé spécifiques aux périodes d'arrêt de tranche programmé des centrales nucléaires de type "réacteur à eau sous pression". Elle débouche sur des questions résiduelles plus générales concernant les relations entre les conditions de travail, l'organisation du travail et la santé. Pour avancer sur le sujet, une approche pluridisciplinaire semble indispensable. Les résultats d'études qualitatives et quantitatives s'enrichissent mutuellement. De nouvelles études qualitatives permettraient de trancher parmi les différentes interprétations proposées des phénomènes observés. Des études ergonomiques permettraient de mieux analyser l'activité et les conditions de travail en arrêt de tranche. Des études de psychodynamique du travail permettraient de mieux appréhender les aspects psychologiques de la santé en contexte professionnel. Des études de psychosociologie des organisations complèteraient l'approche du problème sur ces aspects collectifs.

Sur le plan quantitatif, enfin, un suivi longitudinal des agents enquêtés permettrait d'envisager les conséquences à moyen et à long terme des arrêts de tranche sur la santé non appréciables à partir d'une enquête transversale.

\section{Remerciements}

Les auteurs remercient Ellen Imbernon pour son aide dans la rédaction de l'article et Michèle Stef pour la mise en forme.

\section{RÉFÉRENCES}

[1] BAUMONT G. - Communication personnelle, IPSN, 1994.

[2] DAHLGREN K. - Shift work, work scheduling and their impact on operators in nuclear power plants. IEEE $4^{\text {th }}$ conference on Human factors and power plants, Monterey, CA, June 5-9, 1988. New York : Institute of Electrical and Electronics Engineers.

[3] DEJOURS C. - Plaisir et souffrance dans le travail. Paris: Editions de l'AOCIPE, 1988. 
[4] DEJOURS C. - Travail, usure mentale, essai de psychopathologie du travail - de la psychopathologie du travail à la psychodynamique du travail, Paris : Bayard, 1993.

[5] FUHRER R., ROUILLON F. - La version française de l'échelle CES-D (Center for epidemiological studies-depression scale), description et traduction de l'échelle d'auto-evaluation, Psychiatr. Psychobiol., 1989, 4, 163-166.

[6] HUEZ D. - Approche psychopathologique en médecine du travail chez les agents de maintenance et de conduite d'une industrie de process. In: Actes du $28^{e}$ congrès de la Société d'ergonomie de langue française (SELF), Genève, 22-24 septembre 1993.

[7] KANDAROUN R., HUEZ D. - Le collectif dans les activités de consignation en centrale nucléaire. In: Actes du $27^{\mathrm{e}}$ congrès de la Société d'ergonomie de langue française (SELF), Lille, 23 septembre 1992.

[8] LEWKOWITCH A. - Communication personnelle, EDF, 1994.

[9] SPIELBERGER C.D., VAGG P.R., BARKER L.R., DONHAM G.W., WESTBERRY L.G. - The factor structure of the state and trait anxiety inventory. In: Stress and anxiety, 7 , (I.G. Sarason et C.D. Spielberger, Eds). New York : Hemisphere/Wiley, 1980.

[10] SPIELBERGER C.D. - State and trait anxiety inventory in a comprehensive bibliography. Palo Alto, CA : Consulting psychologists press, 1983. 